\title{
Heritability of Sleep and Its Disorders in Childhood and Adolescence
}

\author{
Katie J. S. Lewis ${ }^{1}$. Alice M. Gregory ${ }^{2}$ \\ Accepted: 7 September 2021 / Published online: 22 November 2021 \\ (c) Crown 2021
}

\begin{abstract}
Purpose of Review This review summarizes recent literature on the heritability of sleep and sleep disorders in childhood and adolescence. We also identify gaps in the literature and priorities for future research.

Recent Findings Findings indicate that age, measurement method, reporter, and timing of sleep measurements can influence heritability estimates. Recent genome-wide association studies (GWAS) have identified differences in the heritability of sleep problems when ancestral differences are considered, but sample sizes are small compared to adult GWAS. Most studies focus on sleep variables in the full range rather than on disorder. Studies using objective measures of sleep typically comprised small samples.

Summary Current evidence demonstrates a wide range of heritability estimates across sleep phenotypes in childhood and adolescence, but research in larger samples, particularly using objective sleep measures and GWAS, is needed. Further understanding of environmental mechanisms and the interaction between genes and environment is key for future research.
\end{abstract}

Keywords Adolescence $\cdot$ Childhood $\cdot$ Circadian rhythms $\cdot$ Heritability $\cdot$ Sleep

\section{Introduction}

In recent years, there have been rapid advances in our understanding of the heritability of sleep phenotypes in humans, but research has primarily focused on adults. Significant developmental changes in sleep physiology and behaviors occur across childhood and adolescence [1-3], and this might have implications for estimates of heritability of sleep across the lifespan. In this review, we provide a brief overview of childhood and adolescent sleep, followed by a summary of methods used to assess sleep and heritability. We then focus on literature published within the last 5 years on the heritability of sleep and its disorders in childhood

This article is part of the Topical Collection on Sleep and Development

Katie J. S. Lewis

lewisk18@ cardiff.ac.uk

1 Division of Psychological Medicine \& Clinical Neurosciences, MRC Centre for Neuropsychiatric Genetics \& Genomics, Cardiff University School of Medicine, Hadyn Ellis Building, Maindy Road, CF24 4HQ Cardiff, UK

2 Department of Psychology, Goldsmiths, University of London, London, UK and adolescence. Finally, we highlight areas of interest for future research.

\section{Sleep and Sleep Disorders in Childhood and Adolescence}

Numerous lines of evidence suggest that sleep is important for children and adolescents, including for their physical and mental health, cognitive processing, and educational attainment $[1,4]$. The dominant theory posits that sleep timing and duration are regulated by two processes [5]: Process $S$, a homeostatic process in which "sleep pressure" increases during waking hours and dissipates during sleep, and Process $C$, circadian (approximately 24-h) peaks and troughs in arousal. Process S and Process $\mathrm{C}$ interact to promote sleep onset when sleep pressure is highest and circadian timing for arousal is low. Research on the neurobiological underpinnings of the two-process model is ongoing, but current evidence suggests that Process $S$ is linked to the accumulation of certain molecules including adenosine in the brain [6] and that Process $\mathrm{C}$ is primarily controlled by the suprachiasmatic nuclei, a collection of neurons in the anterior hypothalamus [7]. 
After sleep onset, in adults, the neurophysiology of sleep can be divided into two main states: non-rapid eye movement (NREM) sleep and rapid eye movement (REM) sleep. NREM sleep typically follows sleep onset, and is divided into 3 stages (N1, N2, and N3) [8]. N1 and N2 are lighter stages of sleep, whereas N3 (also known as deep sleep or slow-wave sleep) is characterized by low-frequency, highamplitude brain activity. K-complexes and sleep spindles (short bursts of 12-14 Hz activity [9]) typically occur in N2. REM sleep typically occurs after progression through stages N1-N3 (and then back to N2). It is characterized by rapid eye movements, muscle paralysis, and high-frequency, low-amplitude brain activity resembling wakefulness, and is the stage of sleep during which vivid dreaming is most likely [10]. Progressing through one cycle of sleep typically takes 50-60 min in infants [11], which then lengthens to 90-110 min in adults and is repeated 4-6 times during the night [12].

Comprehensive accounts of developmental changes in sleep timing and neurophysiology have been reviewed elsewhere $[3,13,14]$. When individuals transition from childhood to adolescence, sleep timing becomes more delayed [15]. This has been linked to puberty onset and is thought to be partly due to sleep pressure accumulating more slowly throughout the day (however, the dissipation of sleep pressure appears to remain the same as in childhood) $[2,14,16]$. Sleep architecture also changes, with reductions in REM sleep from infancy to childhood, and reductions in slowwave sleep and slow-wave activity after the onset of puberty [13]. There are also other psychological and socio-cultural influences on sleep during adolescence, such as greater autonomy over bedtimes and changes in social behavior [16].

\section{Sleep Disorders}

The International Classification of Sleep Disorders (ICSD3) [17] groups sleep disorders under the seven categories (insomnia, sleep-related breathing disorders, central disorders of hypersomnolence, circadian rhythm sleep-wake disorders, sleep-related movement disorders, and other sleep disorders). These are summarized in Table 1.

\section{Measuring Sleep}

Subjective measures of sleep include sleep diaries and questionnaires, which can be collected in both parents/ caregivers and children. Actigraphy is often used as an objective measure of sleep and requires participants to wear a device (typically on their wrist or leg) which detects movement. Algorithms are then used to estimate periods of sleep and wakefulness. These algorithms are validated using the "gold-standard" objective measure of sleep, polysomnography (PSG), in which information from electroencephalography (EEG), electrooculography, and electromyography are combined to determine the presence of the sleep stages described above [1]. Videography and other objective measures are also available [18].

\section{Heritability}

Children differ greatly in terms of their sleep, both for genetic and environmental reasons. Heritability is defined as "the proportion of phenotypic differences among individuals that can be attributed to genetic differences in a particular population" [19]. A heritability estimate is specific to the population from which it was derived, and therefore varies depending on the characteristics of that sample. Heritability for psychological and behavioral traits is rarely (if ever) estimated to be $100 \%$ heritable [20]. This is partly because measurement error is larger for these traits compared to physical traits such as height, but also reflects the influence of the environment.

Common misconceptions about heritability outlined by Visscher et al. [21] are summarized below:

(1) Heritability is the proportion of a phenotype that is passed on to the next generation: Actually, genes are passed on, not phenotypes. This is the most common misconception and is common even amongst those who are educated in genetics [22].

(2) High heritability implies genetic determination: Instead, a high heritability indicates that the phenotype of an individual is a good predictor of genotype. However, due to environmental influences, phenotype is not determined by genotype alone.

(3) Low heritability implies no additive genetic variance: Instead, low heritability means that of all observed variation, a small proportion is caused by variation in genotypes.

(4) Heritability is informative about the nature of betweengroup differences: This is incorrect, actually heritability should not be used to predict changes in a population over time or differences between groups because heritability is defined for a particular population in a particular environment and therefore cannot be applied to other populations which may have different environments.

(5) A large heritability implies genes of a large effect: This is not the case, and it is more common for high heritability estimates to be due to a polygenic effect (multiple genes), which individually explain a low proportion of variance. 
Table 1 Summary of International Classification of Sleep Disorders-3rd Edition (ICSD-3) sleep disorder categories

\section{Category}

Insomnia

Central disorders of hypersomnolence

Sleep-related breathing disorders

Sleep-related movement disorders

Parasomnias
Example disorders

Behavioral insomnia of childhood:

- Difficulty initiating or maintaining sleep despite adequate opportunity

- Poor sleep quality with daytime impairment

- Symptoms present at least three times per week for at least 3 months

Additional specifiers:

- Sleep onset association - child associates their ability to fall asleep with inappropriate environmental stimuli, such as watching television or falling asleep in the car. This means that when that stimulus is absent, the child experiences difficulties falling asleep

- Limit setting insomnia - child refuses to go to bed or employs tactics to stall bedtime

Excessive daytime sleepiness (either prolonged sleep duration or sleepiness) that is not caused by another sleep disorder or circadian rhythm misalignment. Disorders in this section include:

- Narcolepsy - characterized by unintentional sleep bouts, short REM sleep onset latency, and sometimes cataplexy (temporary loss of muscle tone)

- Idiopathic hypersomnia - hypersomnia that cannot be explained by another condition

- Kleine-Levin syndrome — a rare disorder primarily affecting adolescent males [75] characterized by recurrent episodes of excessive sleep, accompanied by "binge eating" and hypersexuality

Abnormal respiration during sleep. Sleep apnea occurs when breathing temporarily stops during sleep, resulting in reduced blood oxygen saturation, sleep fragmentation, and daytime sleepiness. Three types of sleep apnea are:

- Obstructive sleep apnea (OSA) — caused by obstruction of the upper airways. Symptoms of pediatric OSA - snoring, labored/obstructed breathing, or daytime consequences such as sleepiness or hyperactivity to be present

- Central sleep apnea (CSA) - caused by a reduced drive to breath

- Mixed - symptoms of both OSA and CSA

Circadian rhythm sleep-wake disorders (CRSD) Occur when there is a misalignment between the circadian sleep-wake cycle and the external environment (e.g., socially defined timing of school, work, or social activities)

- The misalignment can occur because sleep onset is later (delayed sleep-wake phase disorder) or earlier (advanced sleep-wake phase disorder) than is desired, although other forms of CRSD exist

- The misalignment must be accompanied by insomnia or excessive daytime sleepiness, distress, or impairment, and must last for at least 3 months (with the exception of jet lag disorder) [17]

Movements that prevent or disturb sleep. These disorders include:

- Restless legs syndrome (RLS) - an urge to move the legs that is sometimes accompanied by an uncomfortable sensation. This is partially or totally relieved by movement and occurs primarily in the evening or night

- Periodic limb movement disorder (PMLD) — limb movements that occur during sleep (more than 5 times per hour in children, more than 15 times per hour in adults) which are accompanied by sleep disturbance or other functional impairment

Physical events or experiences occurring during sleep or in the transition to/from sleep. They can be grouped into the following categories:

- NREM parasomnias - also known as disorders of arousal from NREM, these commonly occur during slow-wave sleep (N3) and include sleepwalking, confusional arousals, sleep terrors, and sleep-related eating disorder

- REM parasomnias - include REM sleep behavior disorder, sleep paralysis, and nightmare disorder

- Other parasomnias - occur during either NREM or REM sleep, or during wakefulness soon after sleep. They include exploding head syndrome and sleep enuresis (bed wetting)

NREM parasomnias such as sleepwalking are more common in children than adults and are often considered a normal part of development $[76,77]$. However, the persistence of these parasomnias past a particular age is considered problematic. For example, sleep enuresis is only considered atypical when it persists after 5 years of age

Note. There is an additional section in the ICSD-3 which focuses on sleep disorders that cannot be classified elsewhere 


\section{Family and Adoption Studies}

Family studies sample individuals in a population who have a disorder and assess the risk for the disorder in their relatives. A traditional family design cannot be used to disaggregate genetic from environmental effects because family members usually share both genetic and environmental influences [23, 24]. We will therefore not review studies using this method. In contrast, adoption studies can disaggregate the effects of genetics and environment because they can compare the degree of similarity between people who share different amounts of genetic and environmental similarity (e.g., the adoptees and their adoptive and biological parents).

\section{Twin Studies}

Twin studies can be used to estimate heritability by comparing monozygotic (identical) twins, who share $100 \%$ of their genes, to dizygotic (non-identical) twins, who share, on average, $50 \%$ of their segregating genes. Heritability is estimated by comparing the degree of similarity for a trait (e.g., sleep duration) in monozygotic twin pairs compared to dizygotic twin pairs [25]. Four sources of variance can be estimated: Additive genetic influences ("A"); Dominant (nonadditive) genetic influences ("D"), Common (shared) environmental influences ("C") which make family members more alike; and Non-shared Environmental influences ("E"), which make family members less alike (and includes measurement error) [19]. Using a standard twin design, it is not possible to estimate $\mathrm{D}$ and $\mathrm{C}$ in the same model; therefore, ACE and ADE models are examined separately.

\section{Genome-Wide Association Studies (GWAS)}

GWAS use a case-control method in samples from populations to detect associations between genetic markers (called single-nucleotide polymorphisms, SNPs) and a disease or trait of interest across the genome [26]. From this, SNP heritability $\left(\mathrm{h}^{2}{ }_{\mathrm{SNP}}\right)$ can be estimated, which is the proportion of variance in liability associated with common SNPs genome-wide. There are several methods to estimate SNP heritability from GWAS data, each with different assumptions [27]. In contrast to twin studies, in which heritability represents the proportion of variance due to common and rare genetic variants, SNP heritability represents the proportion of variance due to common genetic variants. This is one reason why SNP heritability is usually lower than heritability estimated from twin studies [27].
Heritability of Sleep and Sleep Disorders in Childhood and Adolescence

\section{Twin Studies}

In recent years, amongst the different techniques used to inform about heritability, twin studies have provided the most information about sleep and sleep disorders in children and adolescence. These studies focus on a plethora of sleep phenotypes.

\section{Sleep Duration}

Kocevska et al. [28••] meta-analyzed data from 19 twin studies ( $N=43,328$, age 6 months to 88 years) assessing sleep duration using self-report, parent reports, actigraphy, and polysomnography. Heritability of sleep duration varied by age, ranging from $17 \%$ in infancy to $69 \%$ in adolescence (46\% across all ages). Heritability estimates also varied according to the method of measuring sleep and by reporter, with lower estimates for parent reports (8\%) vs. self-reports $(38 \%)$ or sleep diary $(52 \%)$. These discrepancies were also found by Breitenstein et al. [29••] who found that the heritability of sleep duration in 8-year-old children $(N=608)$ measured using actigraphy was $81 \%$ compared to $21 \%$ for parent reports.

Inderkum and Tarokh [30] assessed sleep duration for 6 months in 51 adolescents (11-14 years) using actigraphy and self-reports. Heritability estimates for sleep duration measured using actigraphy were $15 \%$ on school days but $68 \%$ on free days (weekends, public holidays, and vacations) and $45 \%$ on holidays (public holidays and vacations). However, self-reported sleep duration was $19 \%$ on school days and $2 \%$ on free days. The more similar estimates between sleep duration on school days as compared to free days could be explained by the fact that correlations between subjective and objective measures were higher on school days (0.53) compared to free days $(0.25)$. The authors hypothesized that sleep duration may be more accurately recalled for school days than free days because bedtimes and risetimes are likely to be influenced by school start times.

\section{Sleep Quality}

Kocesvska et al. [28••] meta-analyzed data from 13 twin studies of sleep quality ( $N=43,328$, age $16-82$ years). Sleep quality was assessed using self-report (primarily using the Pittsburgh Sleep Quality Index [31]). Two of the studies were conducted in adolescents (age 16-18) with heritability estimates ranging from 33 to $41 \%$. The heritability estimate across all ages was $44 \%$, which did not differ significantly 
by age group. This is similar to a recent study of 10,222 16-year-old twins which estimated the heritability of sleep quality to be $42 \%$ [32].

Minutes of wake after sleep onset (WASO) and sleep efficiency (SE) can also be considered markers of sleep quality. Recent heritability estimates for WASO ranged from 73 to $85 \%[30,33]$ and 55 to $80 \%$ for SE [29••, 30]. Inderkum and Tarokh [30] also found that estimates differed depending on whether sleep was measured on school days or free days, but the differences were not as marked as those found for sleep duration. Lower heritability estimates for WASO and SE were observed in a recent EEG study in adolescents, but this had a small sample size $(N=60)$ [33].

\section{Circadian Parameters}

In a study of 608 8-year-olds, Breitenstein et al. [29••] assessed circadian timing and variability of sleep using actigraphy. They did not find evidence that sleep midpoint and sleep midpoint variability were explained by genetic factors, and most of the variance was explained by shared environmental factors. Heritability estimates for chronotype in adolescents (11-14 years) were found to be higher on free days (87-91\%) compared to school days (14\%) when assessed using actigraphy [30], and 76\% when assessed using a chronotype questionnaire. However, the small sample size $(N=51)$ means that there is unlikely to be power to obtain stable estimates. Gehrman and colleagues derived numerous circadian parameters from actigraphy data in 142 twins aged between 16 and 40 years [34•]. They estimated heritability using Sequential Oligogenic Linkage Analysis Routines. Circadian heritability estimates that remained significant after correcting for age and sex were primary minimum phase (88\%), relative amplitude (57\%), and M10 (10 h with maximal activity) (64\%). However, there was a broad age range and estimates were not conducted in adolescents/ young adult samples separately.

One recent study assessed the heritability of biological markers of circadian rhythms. Ouellet-Morin and colleagues [35] examined the cortisol awakening response (CAR), awakening level, and cortisol change from morning to evening (diurnal change) in 592 14-year-old twins. The genetic influence for CAR, awakening level, and diurnal change was estimated at $49.5 \%(\mathrm{~A}+\mathrm{D}), 27.8 \%(\mathrm{~A})$, and $31.4 \%(\mathrm{~A})$, respectively. Using multivariate analysis, the authors found that the influence of A and D for CAR was $39.5 \%$ and $10 \%$, respectively.

\section{Sleep Architecture}

The study by Gehrman et al. [34•] described above also derived duration of "light sleep" and "deep sleep" behavioral states from actigraphy using an algorithm based on second-by-second variability in movement. After correcting for age and sex, heritability for the duration of "light sleep" and "deep sleep" was $41 \%$ and $21 \%$, respectively. Of note, the creators of the algorithm used to define light and deep sleep caution that these behavioral states do not correspond well with PSG-defined sleep stages [36]. Several high-density EEG studies have also been conducted in adolescents. Markovic et al. [37] measured sleep in 50 adolescents (mean age 13.2 years, range $=10-15$ ). They assessed the distribution of EEG power (activity of different EEG frequencies) during NREM and REM sleep. In contrast to findings from adult studies [38], heritability estimates were low for delta $(1-4.6 \mathrm{~Hz})$ and sigma $(11-16 \mathrm{~Hz})$ bands in NREM $\left(0.12 \leq h^{2} \leq 0.2\right)$ and REM sleep $\left(0.01 \leq h^{2} \leq 0.2\right)$. There was higher heritability for beta bands $(16.2-24 \mathrm{~Hz})$ in NREM $\left(0.48 \leq h^{2} \leq 0.51\right)$ and REM sleep $\left(0.52 \leq h^{2} \leq 0.57\right)$. This emphasized the importance of examining high frequency ranges in EEG studies and the effect of shared environmental influences on other frequency bands. Markovic and colleagues [39] also examined the heritability of sleep EEG coherence in 62 adolescents (mean age $=12.5$ years). EEG coherence measures connectivity based on correlation of EEG signals at a specific frequency and is a potential indicator of increased myelination and rewiring of the brain during development [40]. Across frequencies and sleep states, the heritability of sleep EEG coherence was 19\%, with stronger contributions for unique environmental factors (median value range: $45 \% \leq \mathrm{E} \leq 75 \%$ ). The authors found that EEG coherence was strongest for sleep spindles which showed on average a heritability of $48 \%$ across connections. Finally, Rusterholz and colleagues [33] used high-density EEG to estimate the heritability of several sleep parameters in 60 adolescent twins (mean age $=12.46$ years). Most sleep parameters did not show strong genetic influences: number of minutes of REM sleep, sleep efficiency, and total sleep time showed high unique environmental influences $(81 \% \leq \mathrm{E} \leq 98 \%)$, and variance in minutes of stage 1 sleep was primarily influenced by shared environmental factors $(C=85 \%)$. Moderate heritability was observed for duration of slow-wave sleep (37\%), REM sleep latency (40\%), and sleep efficiency (32\%). When assessing EEG power in posterior regions of the brain, the authors found that $80-90 \%$ of the variance in slow oscillations, slow wave, and spindle activity was due to genetic factors. However, EEG power in anterior regions was primarily driven by shared environmental factors.

\section{Insomnia}

In 10,022 16-year-old twins, Madrid-Valero et al. estimated the heritability of insomnia symptoms (assessed using the Insomnia Severity Index) to be $41 \%$ [32]. Shakoor et al. [41] repeated these analyses in a smaller sample from the same 
study $(N=7,442)$ and found that the heritability for insomnia was lower for boys (34\%) than girls (42\%). Madrid-Valero et al. [42] also found that the heritability for Child Behaviour Checklist (CBCL) items "sleeps less than most kids" and "trouble sleeping" was $85 \%$ and $62 \%$, respectively, in 2,060 children (age 8.06 years, range $=6-12$ ). Other twin studies have examined the heritability of sleep onset latency (SOL), which in cases might be relevant to sleep onset insomnia. Recent studies using actigraphy estimate SOL heritability at $30 \%$ at age 8 [29••] and $48-77 \%$ at age 12 [30]. This is within the same range as a recent EEG study in 12-14-yearold adolescents, which estimated SOL heritability at $72 \%$ (although the $N$ in this study was small, $N=60$ ) [33].

\section{Other Sleep-Related Difficulties and Disorders}

Breitenstein et al. [29••] estimated the heritability of daytime sleepiness to be $27 \%$ in 608 children (age 8 years, $S D=0.63$ ) using parent reports. In addition, Champion et al. [43] assessed pediatric restless legs syndrome (RLS) assessed by parent questionnaire and rated against 4 essential criteria defined by the International RLS Study Group in 2,033 twins aged 10.5 years (range 3-18). They found that heritability differed according to RLS subtype, being $14 \%$ for painless RLS but $64 \%$ for painful RLS. Breitenstein et al. [44] assessed sleep problems at age 5 in 406 twins (age 4.8 years, $S D=0.39$ ) using total scores on the Child Sleep Habits Questionnaire [45]. Heritability for the total score was estimated at $28 \%$. As outlined above, Madrid-Valero et al. [42] assessed sleep problems using individual items from the CBCL in 2,060 children (age 8.06 years, range $=6-12$ ). In addition to the aforementioned insomnia-related items, heritability estimates for the other CBCL items were as follows: "sleeps more than most kids" (89\% [ADE model]), "sleeps less than most kids" (85\%) and "overtired" (83\%), "nightmares" (73\%), "talks or walks in sleep" (72\%), and "trouble sleeping" (62\%).

\section{GWAS}

Marinelli et al. [46] conducted a GWAS of sleep duration in 10,554 children aged 2-14 years from the EAGLE consortium. SNP heritability of sleep duration was estimated at $14 \%$ after adjusting for age, sex, and principal components. In 2019, Dashti et al. [47] compared the results of this study with an adult sleep duration GWAS (SNP heritability 9.8\%). They found that none of the 78 genome-wide significant loci identified in the adult GWAS was replicated in the EAGLE GWAS, but 45 of the 77 loci discovered in the EAGLE study showed consistent directionality in the adult GWAS.

Ohi et al. [48••] conducted a GWAS of sleep problems using the total score from the Sleep Disturbance Scale for Children [49] in 9,683 children aged 9-10 years. SNP heritability was $11 \%$ in children of European ancestry and $14 \%$ in children of trans-ancestry [48 • ]. A factor analysis on scores from the SDSC was used to derive subscales representing common sleep disorders. The subscales and corresponding SNP heritability estimates for each subscale are summarized in Table 2, ranging from 2.1 to $22.9 \%$. The GWAS for sleep breathing disorders (sleep apnea and snoring) and sleep hyperhidrosis (falling asleep sweating and night sweating) subscales could not significantly explain variances in these phenotypes.

One other GWAS was conducted by Jørgensen et al. [50••], who assessed lifetime nocturnal enuresis diagnosis in a Danish population using ICD-10 criteria and redeemed desmopressin prescriptions (3,882 cases, 31,073 controls). SNP heritability ranged from 23.9 to $30.4 \%$ assuming a nocturnal enuresis prevalence of $7-15 \%$. This is also the first GWAS to identify genome-wide significant loci for nocturnal enuresis and to identify a genetic overlap with attentiondeficit hyperactivity disorder.

\section{Future Directions}

The studies in this review highlight several key areas for further consideration. First, most research utilized twin studies. Only 3 sleep GWAS in young people were published in the last 5 years and these had much smaller sample sizes compared to adult GWAS. There is evidence that heritability of

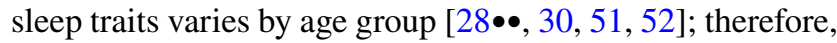
future GWAS should examine genetic influences across different developmental stages, ideally using longitudinal data. Mendelian randomization (MR) studies often use SNPs identified in GWAS to infer causal relationships (e.g., between insomnia and depression). This assumes SNPs index sleep phenotypes occurring before onset of the disorder in question. However, most MR studies use SNPs from UK Biobank GWAS, with participants aged 40-69 years [53]. Existing comparisons between child and adult sleep GWAS suggest different SNPs are associated with sleep traits in childhood and adulthood [47]. It is therefore questionable whether MR studies using SNPs associated with midlife sleep phenotypes can adequately assess causal relationships between sleep and disorders which commonly develop in childhood or adolescence (e.g., depression [54]). This also applies when examining associations between polygenic scores (PGS) for sleep traits and other phenotypes in childhood and adolescence, as PGS are often derived using adult GWAS summary statistics. Furthermore, GWAS often rely on questionnaire measures to assess sleep phenotypes, and the studies reviewed in this paper demonstrate how estimates of genetic influence vary considerably according to measurement. Large sleep GWAS in child and adolescent samples using a range of measurement methods are therefore vital. 


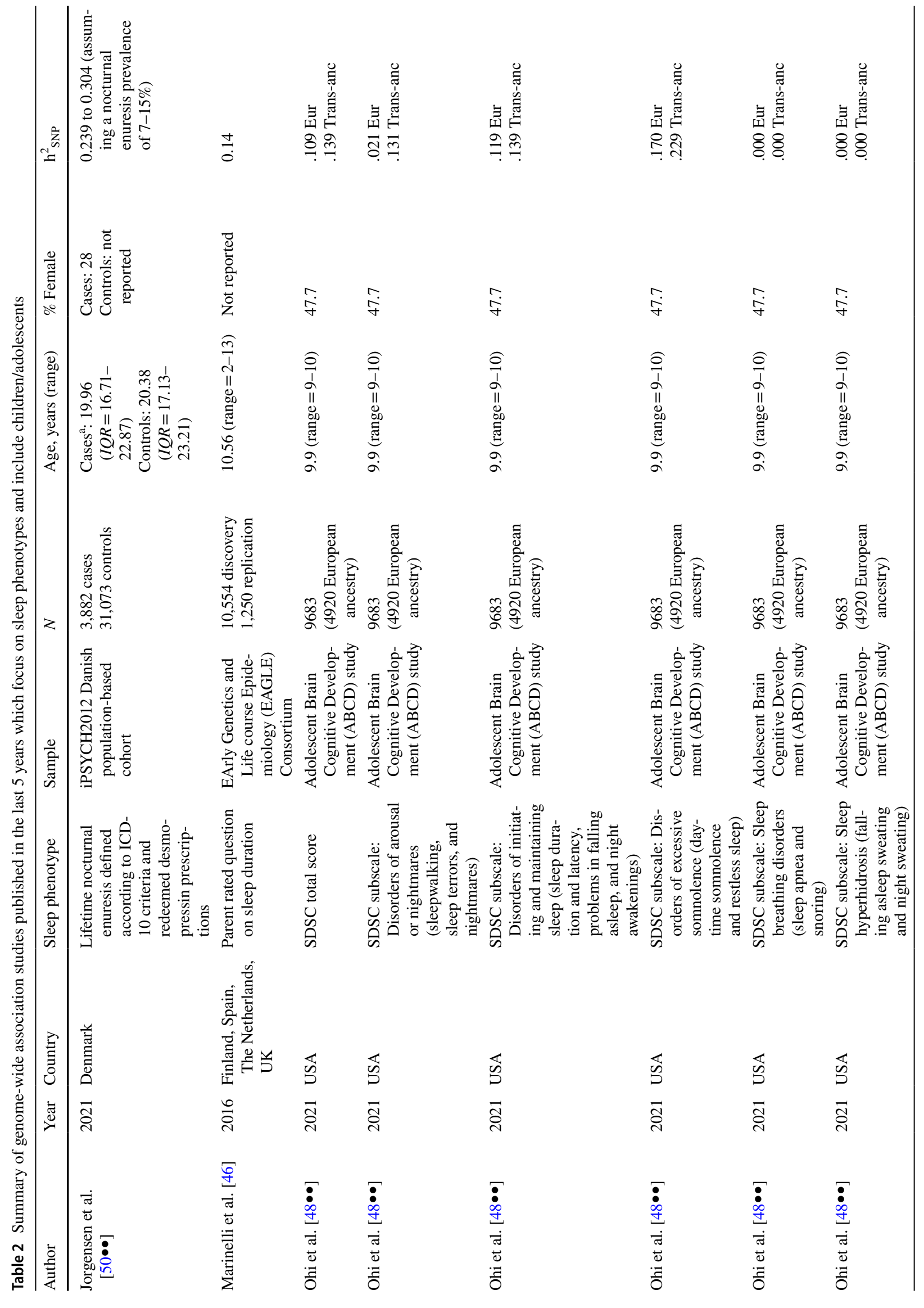




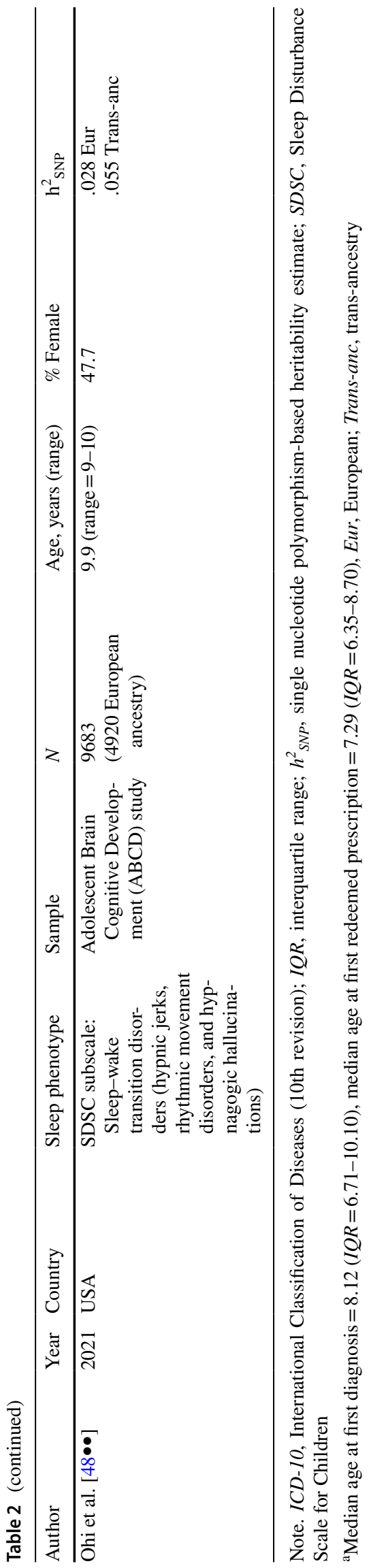

Second, the reviewed studies highlighted several factors to consider in future research. These include ancestral group [48••], whether sleep is measured on weekdays or free days

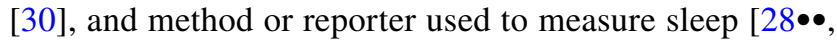
$29 \bullet \bullet]$. However, differences according to measurement method could be driven by unstable estimates, as twin studies using objective measures of sleep often used small sample sizes $(N<150)$ and are likely to be underpowered. Larger studies using objective sleep measures are needed. We also found that only two studies assessed sleep disorders using diagnostic criteria $[43,50 \bullet \bullet]$. Most studies focused on traits related to insomnia, and future research examining other disorders in childhood and adolescence is needed. In addition, few studies reported heritability estimates by gender despite evidence that there are gender differences in sleep EEG characteristics [55] and risk for insomnia [56]. Recent meta-analyses of twin studies have not found evidence that heritability for sleep duration, sleep quality, or insomnia is

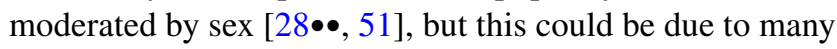
studies not reporting results stratified by gender [51].

\section{Heritability Estimates}

A recent study found that heritability estimates are often misunderstood, even amongst those with genetics training [22]. As our understanding of the role of genetics on sleep progresses, it is increasingly important for researchers to effectively communicate these findings to the public. For example, heritability estimates do not assess the influence of de novo mutations, as by definition they are not inherited, and most studies estimate narrow sense heritability [57] which assumes the absence of gene-gene and geneenvironment interactions. Furthermore, SNP heritability does not consider the impact of rare genetic variants, which may have a greater impact than common variants [58]. These factors and other mechanisms such as epigenetic inheritance [59] may explain why SNP heritability estimates are often much lower than those from twin studies. Some researchers have questioned whether it is possible to separate genetic from environmental influences on complex traits due to the complex way in which genes and environment interact over time and have argued that there should be less focus on refining heritability estimates and more on understanding this complexity [57].

\section{Environmental Influences and Other Mechanisms}

The reviewed studies demonstrate that environmental influences often play a large role in explaining variance in sleep phenotypes. Adoption studies and other genetically sensitive designs can disentangle the effects of environment from inherited factors. A key example is a study by Lewis and colleagues, who examined familial transition 
of depression by analyzing data from children conceived through in vitro fertilization who were either genetically related or unrelated to their parents [60]. They found that the correlations between parent and child depressive symptoms were similar regardless of biological relatedness, suggesting that environmental influences play a role in the intergenerational transmission of depression symptoms. We are not aware of any studies that have used this approach to examine intergenerational transmission of sleep phenotypes, but this provides a fruitful avenue for future work.

Identifying these environmental influences across different ages and sleep phenotypes will be crucial in understanding the aetiology of sleep disorders. There are multiple candidate environmental influences on child and adolescent sleep, including the use of digital media, diet/caffeine intake, parent-imposed bedtimes and sleep habits, and psychosocial changes during adolescence $[2,16,61,62]$. Future research could examine genetic influences on these "environmental traits." Recent studies have implicated other mechanisms such as bullying [41], sibling conflict [44], internalizing problems [32], antisocial behavior [42], emotional regulation [63], and impulsivity and anger [64]. There have also been studies in young adults that have examined pre-sleep arousal [65], dysfunctional beliefs about sleep [66], and loneliness [67] as other pathways that affect sleep. Future work could explore how these factors influence sleep in younger populations.

Environmental factors can also change heritability estimates over time, even for traits with a high genetic influence [68], and this should be considered in future research. Key factors to consider are increases in the use of digital technology, lifestyle changes due to the COVID-19 pandemic (e.g., more indoor activities and reduced sunlight exposure) [69], and long-term sleep disturbances arising from contracting COVID-19 [70]. Understanding gene-environment interactions and epigenetic influences will also be important. For example, insomnia is thought to develop because individuals with a predisposition to insomnia are more likely to develop insomnia after precipitating factors (e.g., stress) [71]. Palagini and colleagues hypothesized that epigenetic mechanisms may predispose individuals to insomnia, either through early life experiences or transgenerational epigenetic inheritance, and may also be involved in the maintenance of insomnia [72]. Epigenetic influences have also been implicated in other sleep disorders [73]. A recent study in 10-year-old children found that sleep duration measured using actigraphy was associated with DNA methylation patterns in a region of the genome implicating genes previously identified in sleep GWAS [74•]. Understanding how epigenetic mechanisms influence sleep in childhood and adolescence could be a promising avenue to inform our understanding of sleep and sleep disorders.

\section{Conclusion}

Our review of recent literature highlights that more research is needed to understand how genetic factors influence sleep and sleep disorders across different ages in childhood and adolescence. This is particularly the case for GWAS, which are primarily conducted in adults. Further understanding of how genes interact with the environment and other psychological and biological mechanisms will be crucial to inform our understanding of the aetiology of sleep disorders and inform interventions to improve sleep in young people.

Funding Katie Lewis was supported by Health and Care Research Wales and the Wellcome Trust (Grant No: 220488/Z/20/Z).

\section{Declarations}

Conflict of Interest Alice Gregory is an advisor for a project initially sponsored by Johnson's Baby. She receives royalties for two books Nodding Off (Bloomsbury Sigma, 2018) and The Sleepy Pebble (Flying Eye, 2019). She has another contract with Lawrence King Publishers. She is a regular contributor to BBC Focus magazine and has contributed to other outlets (such as The Conversation, The Guardian, and Balance Magazine). She occasionally receives sample products related to sleep (e.g., blue light blocking glasses) and has given a paid talk to a business. Katie Lewis declares no conflicts of interest.

Human and Animal Rights and Informed Consent This article does not contain any studies with human or animal subjects performed by any of the authors.

Open Access This article is licensed under a Creative Commons Attribution 4.0 International License, which permits use, sharing, adaptation, distribution and reproduction in any medium or format, as long as you give appropriate credit to the original author(s) and the source, provide a link to the Creative Commons licence, and indicate if changes were made. The images or other third party material in this article are included in the article's Creative Commons licence, unless indicated otherwise in a credit line to the material. If material is not included in the article's Creative Commons licence and your intended use is not permitted by statutory regulation or exceeds the permitted use, you will need to obtain permission directly from the copyright holder. To view a copy of this licence, visit http://creativecommons.org/licenses/by/4.0/.

\section{References}

Papers of particular interest, published recently, have been highlighted as:

- Of importance

$\bullet$ Of major importance

1. Tarokh L, Saletin JM, Carskadon MA. Sleep in adolescence: physiology, cognition and mental health. Neurosci Biobehav Rev. 2016;70:182-8. 
2. Crowley SJ, Wolfson AR, Tarokh L, Carskadon MA. An update on adolescent sleep: new evidence informing the perfect storm model. J Adolesc. 2018;67:55-65.

3. Barclay NL, Gregory AM (2013) Sleep in childhood and adolescence: age-specific sleep characteristics, common sleep disturbances and associated difficulties. In: The neurobiology of childhood. pp 337-365

4. Gregory AM, Sadeh A. Sleep, emotional and behavioral difficulties in children and adolescents. Sleep Med Rev. 2012;16:129-36.

5. Borbély AA. A two process model of sleep regulation. Hum Neurobiol. 1982;1:195-204.

6. Borbély AA, Daan S, Wirz-Justice A, Deboer T. The twoprocess model of sleep regulation: a reappraisal. J Sleep Res. 2016;25:131-43.

7. Foster RG, Kreitzman L. The rhythms of life: what your body clock means to you! Exp Physiol. 2014;99:599-606.

8. Iber C, Ancoli-Israel S, Chesson A, Quan SF (2007) The AASM manual for the scoring of sleep and associated events: rules, terminology, and technical specification., 1st ed. Westchester, IL

9. Avidan AY (2005) Recognition of sleep stages and adult scoring technique. Atlas of Sleep Medicine 95-121

10. Krause AJ, Ben Simon E, Mander BA, Greer SM, Saletin JM, Goldstein-Piekarski AN, Walker MP. The sleep-deprived human brain. Nature Publishing Group. 2017;18:404-18.

11. Carskadon MA, Dement WC (2005) Normal human sleep: an overview. Principles and Practice of Sleep Medicine. https://doi. org/10.1016/B0-72-160797-7/50009-4

12. Luyster FS, Strollo PJ, Zee PC, Walsh JK. Sleep: a health imperative. Sleep. 2012;35:727-34.

13. Skeldon A, Derks G, Dijk D-J. Modelling changes in sleep timing and duration across the lifespan: changes in circadian rhythmicity or sleep homeostasis? Sleep Med Rev. 2015;28:92-103.

14. Tarokh L, Short M, Crowley SJ, Fontanellaz-Castiglione CEG, Carskadon MA. Sleep and circadian rhythms in adolescence. Curr Sleep Med Rep. 2019;5:181-92.

15. Roenneberg T, Kuehnle T, Pramstaller PP, Ricken J, Havel M, Guth A, Merrow M. A marker for the end of adolescence. Curr Biol. 2004;14:R1038-9.

16. Carskadon MA. Sleep in adolescents: the perfect storm. Pediatr Clin North Am. 2011;58:637-47.

17. American Academy of Sleep Medicine (2014) International classification of sleep disorders, 3rd ed. American Academy of Sleep Medicine, Darien, IL

18. Sadeh A (2015) Sleep assessment methods. Monographs of the Society for Research in Child Development 33-48

19. Plomin R, DeFries JC, McClearn GE, McGuffin P (2008) Behavioral genetics, 5th ed. Worth, New York, NY

20. Plomin R, DeFries JC, Knopik VS, Neiderhiser JM. Top 10 replicated findings from behavioral genetics. Perspect Psychol Sci. 2016;11:3-23.

21. Visscher PM, Hill WG, Wray NR. Heritability in the genomics era-concepts and misconceptions. Nat Rev Genet. 2008;9:255-66.

22. Madrid-Valero JJ, Chapman R, Bailo E, Ordoñana JR, Selita F, Kovas Y, Gregory AM. What do people know about the heritability of sleep? Behav Genet. 2021;51:144-53.

23. Shih RA, Belmonte PL, Zandi PP. A review of the evidence from family, twin and adoption studies for a genetic contribution to adult psychiatric disorders. Int Rev Psychiatry. 2004;16:260-83.

24. Kendler KS, Neale MC. "Familiality" or heritability. Arch Gen Psychiatry. 2009;66:452.

25. Flint J, Greenspan RJ, Kendler KS (2010) How genes influence behavior, 1st ed. Oxford University Press, New York, NY
26. Corvin A, Craddock N, Sullivan PF. Genome-wide association studies: a primer. Psychol Med. 2010;40:1063-77.

27. Baselmans BML, Yengo L, van Rheenen W, Wray NR. Risk in relatives, heritability, SNP-based heritability, and genetic correlations in psychiatric disorders: a review. Biol Psychiat. 2021;89:11-9.

28.• Kocevska D, Barclay NL, Bramer WM, Gehrman PR, van Someren EJW (2021) Heritability of sleep duration and quality: a systematic review and meta-analysis. Sleep Medicine Reviews 59:101448. This study reports results from two large metaanalyses of twin studies on sleep duration $(N=45,328)$ and sleep quality $(N=39,020)$. The authors found evidence that heritability estimates for sleep duration are moderated by age. They also highlight the need for more research using objective measures of sleep and assessing sleep quality in pediatric populations.

29•• Breitenstein RS, Doane LD, Lemery-Chalfant K (2021) Children's objective sleep assessed with wrist-based accelerometers: strong heritability of objective quantity and quality unique from parent-reported sleep. Sleep 44:1-17. Twin study in $\mathbf{6 0 8}$ children which found that heritability estimates for sleep duration and quality were higher when using actigraphy compared to parent reports. This demonstrates the importance of using multiple measures of sleep, as well as multiple reporters, when assessing genetic influences on children's sleep.

30. Inderkum AP, Tarokh L. High heritability of adolescent sleepwake behavior on free, but not school days: a long-term twin study. Sleep. 2018;41:1-9.

31. Buysse DJ, Reynolds CF, Monk TH, Berman SR, Kupfer DJ. The Pittsburgh Sleep Quality Index: a new instrument for psychiatric practice and research. Psychiatry Res. 1989;28:193-213.

32. Madrid-Valero JJ, Ronald A, Shakeshaft N, Schofield K, Malanchini M, Gregory AM. Sleep quality, insomnia, and internalizing difficulties in adolescents: insights from a twin study. Sleep. 2020;43:1-9.

33. Rusterholz T, Hamann C, Markovic A, Schmidt SJ, Achermann $\mathrm{P}$, Tarokh L. Nature and nurture: brain region-specific inheritance of sleep neurophysiology in adolescence. J Neurosci. 2018;38:9275-85.

34. Gehrman PR, Ghorai A, Goodman M, McCluskey R, Barilla H, Almasy L, Roenneberg T, Bucan M (2019) Twin-based heritability of actimetry traits. Genes, Brain and Behavior e12569. Study of 142 twins using actigraphy to derive novel sleep and circadian parameters that will be useful in future genetic research.

35. Ouellet-Morin I, Brendgen M, Girard A, Lupien SJ, Dionne G, Vitaro F, Boivin M. Evidence of a unique and common genetic etiology between the CAR and the remaining part of the diurnal cycle: a study of 14 year-old twins. Psychoneuroendocrinology. 2016;66:91-100.

36. McCloskey R, Goodman MH, McHugh Grant S, Ghorai A, Gehrman PR, Bućan M (2018) PennZzz - an algorithm for estimating behavioral states from wrist-worn accelerometery. bioRxiv. https://doi.org/10.1101/347807

37. Markovic A, Achermann P, Rusterholz T, Tarokh L. Heritability of sleep EEG topography in adolescence: results from a longitudinal twin study. Sci Rep. 2018;8:1-13.

38. de Gennaro L, Marzano C, Fratello F, et al. The electroencephalographic fingerprint of sleep is genetically determined: a twin study. Ann Neurol. 2008;64:455-60.

39. Markovic A, Kaess M, Tarokh L. Environmental factors shape sleep EEG connectivity during early adolescence. Cereb Cortex. 2020;30:5780-91. 
40. Tarokh L, Carskadon MA, Achermann P. Developmental changes in brain connectivity assessed using the sleep EEG. Neuroscience. 2010;171:622-34.

41. Shakoor S, M.S Zavos H, Gregory AM, Ronald A (2021) The association between bullying-victimisation and sleep disturbances in adolescence: evidence from a twin study. J Sleep Res. https://doi.org/10.1111/jsr.13321

42. Madrid-Valero JJ, Ordoñana JR, Klump KL, Burt SA. Children sleep and antisocial behavior: differential association of sleep with aggression and rule-breaking. J Abnorm Child Psychol. 2019;47:791-9.

43. Champion D, Bui M, Aouad P, et al. Contrasting painless and painful phenotypes of pediatric restless legs syndrome: a twin family study. Sleep Med. 2020;75:361-7.

44. Breitenstein RS, Doane LD, Clifford S, Lemery-Chalfant K. Children's sleep and daytime functioning: increasing heritability and environmental associations with sibling conflict. Soc Dev. 2018;27:967-83.

45. Owens JA, Spirito A, McGuinn M. The Children's Sleep Habits Questionnaire (CSHQ): psychometric properties of a survey instrument for school-aged children. Sleep. 2000;23:1043-51.

46. Marinelli M, Pappa I, Bustamante M, et al. Heritability and genome-wide association analyses of sleep duration in children: the EAGLE consortium. Sleep. 2016;39:1859-69.

47. Dashti HS, Jones SE, Wood AR, et al. Genome-wide association study identifies genetic loci for self-reported habitual sleep duration supported by accelerometer-derived estimates. Nat Commun. 2019;10:1100.

48.• Ohi K, Ochi R, Noda Y, Wada M, Sugiyama S, Nishi A, Shioiri T, Mimura M, Nakajima S (2021) Polygenic risk scores for major psychiatric and neurodevelopmental disorders contribute to sleep disturbance in childhood: Adolescent Brain Cognitive Development (ABCD) study. Translational Psychiatry 11:1-11. This study of $\mathbf{9 , 6 8 3}$ children found that polygenic risk for attention-deficit/hyperactivity disorder and major depressive disorder was associated with sleep disturbances at age 9-10. The authors also conduct several GWAS of sleep phenotypes.

49. Bruni O, Ottaviano S, Guidetti V, Romoli M, Innocenzi M, Cortesi F, Giannotti F. The Sleep Disturbance Scale for Children (SDSC) construction and validation of an instrument to evaluate sleep disturbances in childhood and adolescence. $\mathbf{J}$ Sleep Res. 1996;5:251-61.

$50 . \bullet$ Jørgensen CS, Horsdal HT, Rajagopal VM, et al (2021) Identification of genetic loci associated with nocturnal enuresis: a genome-wide association study. The Lancet Child and Adolescent Health 5:201-209. This is the first GWAS of nocturnal enuresis $(N=34,955)$. The authors identify two loci associated with nocturnal enuresis, which implicated several genes potentially involved in regulating sleep, urine production, and bladder function. They also found that polygenic risk scores for attention-deficit/hyperactivity disorder were associated with nocturnal enuresis.

51. Madrid-Valero JJ, Rubio-Aparicio M, Gregory AM, SánchezMeca J, Ordoñana JR (2021) The heritability of insomnia: systematic review and meta-analysis of twin studies. Sleep Medicine Reviews. https://doi.org/10.1016/j.smrv.2021.101437

52. Barclay NL, Gehrman PR, Gregory AM, Eaves LJ, Silberg JL. The heritability of insomnia progression during childhood/ adolescence: results from a longitudinal twin study. Sleep. 2015;38:109-18.

53. Sudlow C, Gallacher J, Allen N, et al. UK Biobank: an open access resource for identifying the causes of a wide range of complex diseases of middle and old age. PLoS Med. $2015 ; 12: 1-10$.
54. Thapar A, Collishaw S, Pine DS, Thapar AK. Depression in adolescence. The Lancet. 2012;379:1056-67.

55. Markovic A, Kaess M, Tarokh L (2020) Gender differences in adolescent sleep neurophysiology: a high-density sleep EEG study. Scientific Reports 2020 10:1 10:1-13

56. Zhang B, Wing Y-K. Sex differences in insomnia: a metaanalysis. Sleep. 2006;29:85-93.

57. Génin E (2019) Missing heritability of complex diseases: case solved? Human Genetics 2019 139:1 139:103-113

58 Saint Pierre A, Génin E. How important are rare variants in common disease? Brief Funct Genomics. 2014;13:353-61.

59. Trerotola M, Relli V, Simeone P, Alberti S. Epigenetic inheritance and the missing heritability. Hum Genomics. 2015;9:17.

60. Lewis G, Rice F, Harold GT, Collishaw S, Thapar A. Investigating environmental links between parent depression and child depressive/anxiety symptoms using an assisted conception design. J Am Acad Child Adolesc Psychiatry. 2011;50:451.

61. LeBourgeois MK, Hale L, Chang A-M, Akacem LD, Montgomery-Downs HE, Buxton OM. Digital media and sleep in childhood and adolescence. Pediatrics. 2017;140:S92-6.

62. Hale L, Li X, Hartstein LE, LeBourgeois MK. Media use and sleep in teenagers: what do we know? Curr Sleep Med Rep. 2019;5:128-34.

63. Medda E, Alessandri G, Delfino D, Fagnani C, Ferri M, Violani C, Stazi MA. Adolescents self-reported sleep quality and emotional regulation: a discordant twin study. Annali dell'Istituto superiore di sanita. 2011;55:118-23.

64. Miadich SA, Shrewsbury AM, Doane LD, Davis MC, Clifford S, Lemery-Chalfant K. Children's sleep, impulsivity, and anger: shared genetic etiology and implications for developmental psychopathology. J Child Psychol Psychiatry. 2020;61:1070-9.

65. Schneider MN, Denis D, Buysse DJ, Kovas Y, Gregory AM. Associations between pre-sleep arousal and insomnia symptoms in early adulthood: a twin and sibling study. Sleep. 2019;42:1-10.

66. Schneider MN, Kovas Y, Gregory AM. Dysfunctional beliefs about sleep and insomnia symptoms in early adulthood: a twin and sibling study. J Sleep Res. 2019;28:1-11.

67. Matthews T, Danese A, Gregory AM, Caspi A, Moffitt TE, Arseneault L. Sleeping with one eye open: loneliness and sleep quality in young adults. Psychol Med. 2017;47:2177-86.

68. Rutter M, Silberg J, O'Connor T, Simonoff E. Genetics and child psychiatry: I. Advances in quantitative and molecular genetics. J Child Psychol Psychiatry. 1999;40:3-18.

69. Becker SP, Dvorsky MR, Breaux R, Cusick CN, Taylor KP, Langberg JM. Prospective examination of adolescent sleep patterns and behaviors before and during COVID-19. Sleep. 2021;2021:1-11.

70. Huang C, Huang L, Wang Y, et al. 6-month consequences of COVID-19 in patients discharged from hospital: a cohort study. The Lancet. 2021;397:220-32.

71. Spielman AJ, Caruso LS, Glovinsky PB. A behavioral perspective on insomnia treatment. Psychiatr Clin North Am. 1987; 10:541-53.

72. Palagini L, Biber K, Riemann D. The genetics of insomnia-evidence for epigenetic mechanisms? Sleep Med Rev. 2014;18:225-35.

73. Morales-Lara D, De-la-Peña C, Murillo-Rodríguez E (2017) Dad's snoring may have left molecular scars in your DNA: the emerging role of epigenetics in sleep disorders. Molecular Neurobiology 2017 55:4 55:2713-2724 
74. Koopman-Verhoeff ME, Mulder RH, Saletin JM, Reiss I, Horst GTJ van der, Felix JF, Carskadon MA, Tiemeier H, Cecil CAM (2020) Genome-wide DNA methylation patterns associated with sleep and mental health in children: a population-based study. Journal of Child Psychology and Psychiatry 61:1061-1069. Study moving beyond heritability to try to understand more about the links between DNA methylation patterns and sleep.

75. Gadoth N, Oksenberg A. Kleine-Levin syndrome: an update and mini-review. Brain Develop. 2017;39:665-71.
76. Bassetti CL (2015) Sleepwalking: dissociation between "body sleep" and "mind sleep." The Neurology of Consciousness: Cognitive Neuroscience and Neuropathology 129-138

77. Mason TBA, Pack AI (2008) Pediatric parasomnias. In: Sleep in Children. CRC Press, pp 253-272

Publisher's Note Springer Nature remains neutral with regard to jurisdictional claims in published maps and institutional affiliations. 